\title{
Desarrollo y validación de una escala para la medición de la actitud hacia la lactancia materna
}

\author{
Development and validation of a scale for the measurement of attitude \\ towards breastfeeding \\ Desenvolvimento e validação de uma escala para medir a atitude em relação ao \\ aleitamento materno
}

\author{
Álvaro Jácome Orozco 1* , Fabio Alexander Sierra Matamoros², Laura Camila Lozano Neira²,3
}

Recibido: 14 de noviembre de 2020. Aceptado para publicación: 19 de marzo de 2021.

Publicado en línea: 6 de abril de 2021.

https://doi.org/10.35454/rncm.v4n3.234

\section{Resumen}

Introducción: un mejor conocimiento de las mujeres lactantes, para el diseño de estrategias que fomenten la lactancia materna exclusiva, permitirá mejorar las tasas actuales. El uso de instrumentos de medición en conceptos como la actitud frente a la lactancia permiten este propósito. El objetivo del estudio fue desarrollar y validar una escala que mida la actitud frente a la lactancia.

Métodos: se definieron 17 ítems tipo Likert. Se aplicó la escala a 518 madres con productos sanos, a término y en el posparto inmediato, en dos instituciones de Bogotá. Los resultados se evaluaron mediante la teoría clásica de prueba y teoría respuesta del ítem mediante un modelo de crédito parcial.

Resultado: se encontró una validez semántica adecuada. El análisis factorial exploratorio y del contenido de los ítems definió un solo constructo: la actitud. Los índices de consistencia interna y confiabilidad fueron moderados. Los estadísticos de ajuste infit y outfit permitieron conservar todos los ítems en la escala. Las preguntas que más aportaron a la medición evaluaron el conocimiento sobre los beneficios de la lactancia y el uso de leches de fórmula. La escala posee ítems para medir los niveles bajos y medios de actitud, pero requiere otros para discriminar niveles

\section{Summary}

Introduction: Better knowledge of breastfeeding women for the design of strategies that promote exclusive breastfeeding will improve current rates. The use of tools to measure things such as attitude towards breastfeeding contributes to this purpose. The objective of the study was to develop and validate a scale that measures attitude towards breastfeeding.

Method: 17 Likert-type items were defined. The scale was applied to 518 mothers with healthy term newborns in the immediate postpartum period, in two institutions in Bogotá. The results were assessed using the Classical Test Theory and Item Response Theory, with a partial credit model.

Results: The scale has adequate validity. A single construct was defined based on the exploratory factor and item content analysis: attitude. The internal consistency and reliability indices were moderate. The infit and outfit adjustment statistics allowed us to keep all the items on the scale. The questions that contributed the most to the measurement were the ones that evaluated knowledge about the benefits of breastfeeding and the use of milk formula. The scale has items to measure low and medium levels of attitude, but is in need of others to discriminate high levels. The information function showed

\section{Resumo}

Introdução: um melhor conhecimento das mulheres lactantes para o desenho de estratégias que promovam o aleitamento materno exclusivo irá melhorar os índices atuais. A utilização de instrumentos de medição em conceitos como a atitude em relação à amamentação permite este propósito. O objetivo do estudo foi desenvolver e validar uma escala para medir a atitude em relação ao aleitamento materno.

Método: foram definidos 17 itens do tipo Likert. A escala foi aplicada a 518 mães com produtos saudáveis, a termo, no pós-parto imediato, em duas instituições de Bogotá. Os resultados foram avaliados por meio da Teoria Clássica do Teste e da Teoria de Resposta ao item por meio de um modelo de crédito parcial.

Resultados: foi encontrada uma validade semântica adequada. A análise fatorial exploratória e o conteúdo dos itens definiram um único construto: a atitude. Os índices de consistência interna e confiabilidade foram moderados. As estatísticas de ajuste de infit e outfit permitiram manter todos os itens da escala. As questões que mais contribuíram para a medição avaliaram o conhecimento sobre os benefícios da amamentação e da utilização de fórmulas infantis. A escala tem itens para medir níveis baixos e médios de atitude, 
altos. La función de información mostró que la escala es adecuada para medir los niveles medios y bajos de actitud.

Conclusión: la escala desarrollada para la actitud hacia la lactancia materna es válida para discriminar el constructo en pacientes con niveles bajos y medios, lo que muestra su utilidad para identificar madres que se beneficiarían de apoyo en esta actividad.

Palabras clave: actitud, lactancia materna, escalas. that the scale is adequate to measure medium and low levels of attitude.

Conclusions: The scale developed to measure attitude towards breastfeeding is valid to discriminate the construct in patients with low and medium levels, showing its usefulness to identify mothers who would benefit from support in this activity.

Keywords: Attitude; Breastfeeding; Scales. mas requer outros itens que discriminem níveis altos. A função de informação mostrou que a escala é adequada para medir níveis médios e baixos de atitude.

Conclusões: a escala desenvolvida para a atitude frente ao aleitamento materno é válida para discriminar o construto em pacientes com níveis baixos e médios, mostrando sua utilidade para identificar mães que se beneficiariam de apoio em esta atividade.

Palavras-chave: atitude, aleitamento materno, escalas.

\footnotetext{
Departamento de Pediatría. Clínica del Country, Bogotá, Colombia.

2 Fundacion Universitaria de Ciencias de la Salud, Bogotá, Colombia.

3 Hospital de San José de Bogotá, Bogotá, Colombia.
}

* Correspondencia: Álvaro Jácome Orozco. alvarjacome@gmail.com

\section{DECLARACIÓN DE RELEVANCIA CLÍNICA}

Uso de la escala para un diseño de estrategias de promoción de lactancia, de acuerdo con las características culturales de la población a atender, según el grado de conocimiento y actitud frente a la lactancia, previa aplicación de la escala en la población. Asimismo, sirve para medir el impacto de las estrategias aplicadas.

\section{INTRODUCCIÓN}

Sobre la lactancia materna, la Organización Mundial de la Salud (OMS) dice que "es el mejor alimento para los recién nacidos desde el primer momento y de forma exclusiva hasta los 6 meses de edad y complementada hasta más allá de los 2 años”(1). El desarrollo y la validación de escalas se dan a partir de la necesidad de cuantificar hechos no tangibles, como la inteligencia, la calidad de vida, la actitud o la aptitud. A través de las respuestas dadas a los ítems de las escalas o pruebas se determina la magnitud del constructo que se está midiendo $^{(2)}$. Se han elaborado diferentes metodologías para desarrollar escalas, entre las cuales está la teoría clásica de prueba y la teoría de respuesta al ítem ${ }^{(3,4)}$.

En la teoría clásica de prueba, la evaluación del constructo se da a partir de la sumatoria de los ítems que conforman la escala. La puntuación varía desde cero hasta la máxima en la prueba. Está conformada por las respuestas de cada uno de los participantes más el error de medida, lo que ayuda a poder discriminar ítems fáciles y difíciles, y la discriminación de las respuestas. Dentro de las debilidades que se tiene al validar una escala con esta metodología está la dependencia de la población en la que se realiza ${ }^{(4,5)}$.

La teoría de respuesta al ítem utiliza modelos probabilísticos, que relacionan el constructo medido (por ejemplo, la actitud de la madre hacia la lactancia) ${ }^{(6)} \mathrm{y}$ la probabilidad de respuesta a un ítem específico ${ }^{(7)}$. De esta manera, se puede establecer qué tan probable un sujeto responde de manera adecuada a dicho ítem, dado por la cantidad de constructo que tenga el sujeto.

Se reconoce en la actitud y la autoeficacia materna aspectos determinantes en la mujer que lacta, especialmente en las primeras semanas de inicio de la lactancia. Teniendo en cuenta el papel que estas actitudes tienen, se considera necesario cuantificar estas condiciones para evaluar resultados en la implementación de estrategias en salud pública relacionadas con el tema.

Actualmente existen escalas psicométricas relacionadas con el tema. Para la actitud existe la Gender-Role Attitudes Toward Breast-Feeding Scale (GRABS) ${ }^{(8)}$ y la escala Iowa Infant Feeding Attitude Scale (IIFAS) ${ }^{(9)}$. La primera mide la aptitud en relación con la lactancia y mujeres primíparas; mientras que la segunda se validó para medir dos constructos: actitud y conocimiento sobre la lactancia. De ella existen varias adaptaciones culturales $^{(10-12)}$, siendo una escala reconocida. Existen escalas para medir y evaluar la actitud materna frente a la lactancia, con traducciones validadas al español ${ }^{(13,14)}$. 
Sin embargo, estas escalas incluyen ítems que miden aspectos relacionados con el uso de las leches de fórmula, pero no consideran aspectos como la actitud hacia la lactancia exclusiva en los primeros 6 meses, la vinculación de la pareja en la lactancia, las redes de apoyo social y la posibilidad de extracción de leche en madres que trabajan; por esta razón, se decidió construir una escala usando como base el contenido sugerido por la IIFAS ${ }^{(9)}$, e incluir estos nuevos aspectos.

\section{METODOLOGÍA}

\section{Diseño}

El estudio se realizó en dos fases: construcción y validación de la escala.

\section{Construcción de la escala}

Para construir los ítems se realizó una revisión de la literatura sobre la actitud hacia la lactancia materna y las escalas para su medición. A partir de los resultados obtenidos, un pediatra con conocimiento en lactancia materna propuso un grupo de ítems que se sometieron a un proceso iterativo de validación semántica y ajustes por un grupo de expertos, conformado por un gastroenterólogo pediatra, un nutriólogo pediatra, un psicólogo experto en semántica, una enfermera experta en lactancia y una madre. La validación semántica consistió en evaluar la suficiencia, claridad, coherencia y relevancia del ítem. "Suficiencia" indica si los ítems que pertenecen a una misma dimensión bastan para obtener la medición de la misma; "coherencia" evalúa si el ítem tiene relación lógica con la dimensión que se está midiendo; "relevancia" se refiere a si el ítem es esencial o importante, es decir, si debe ser incluido; y "claridad" indica si el ítem se comprende fácilmente, es decir, si su sintáctica y semántica es adecuada. Se empleó una escala de cuatro puntos:

- No cumple con el criterio

- Nivel bajo de cumplimiento

- Nivel moderado de cumplimiento

- Nivel alto de cumplimiento.

Se hicieron ajustes cuando el nivel de cumplimiento fue 3 , al promediar la valoración realizada por los cuatro expertos. Para la clasificación del constructo se empleó una escala de Likert, que es un instrumento psicométrico, donde el encuestado debe indicar su acuerdo o desacuerdo sobre una afirmación, ítem o reactivo, empleando una escala ordenada y unidimensional ${ }^{(15)}$.

\section{Validación de la escala}

Se aplicó la escala a 518 madres de dos instituciones de Bogotá, Colombia, quienes estaban en el puerperio inmediato y en alojamiento conjunto con su hijo. Esta muestra se empleó para evaluar la consistencia interna de la escala, la validez del contenido y para hacer un análisis según la teoría de respuesta al ítem. A 100 madres se le aplicó la escala IIFAS de forma simultánea para evaluar la validez del constructo convergente, mientras que a 55 de ellas se les realizó una nueva aplicación 1 semana después para evaluar la confiabilidad del examen y repetición del examen.

La consistencia interna se evaluó por medio del Alpha de Cronbach estadístico. La validez del contenido se valoró por medio del análisis factorial exploratorio. Se aplicó la prueba de esfericidad de Bartlett y la prueba de Kaiser-Meyer-Olkin para evaluar si la matriz era factorizable; luego se calculó una matriz de correlaciones policóricas para realizar el análisis factorial, teniendo en cuenta que los ítems se construyeron en una escala ordinal; se determinó el número de factores por medio de la inspección del gráfico de sedimentación y los valores propios mayores de 1 . La validez de constructo convergente se evaluó mediante un coeficiente de Spearman y la confiabilidad del examen y repetición del examen, por medio del coeficiente de Lin. Se utilizó el software STATA $15^{\oplus}$ para realizar estos análisis.

El análisis de teoría de respuesta al ítem se llevó a cabo mediante un modelo de Rasch sobre el crédito parcial para analizar la confiabilidad de ítems y personas $^{(16)}$, el ajuste de los ítems en la escala, evaluar la escala de medición de cada ítem y para obtener una estimación de la precisión de la medición de la escala. La confiabilidad de las personas e ítems se midió con índices de confiabilidad (varían entre 0 y 1 , valores más altos implican mejores resultados) y de separación (se sugieren valores mayores de 2 para mostrar una adecuada discriminación). El ajuste se midió con los estadísticos infit y oufit. En su forma sin estandarizar (MNSQ), los valores mayores de 1,5 indican desajuste, mientras que los valores menores de 0,5 muestran redundancia. Por otro lado, en su forma estandarizada (ZSTD), los valores mayores de 1,9 sugieren desajuste, y menores de -1,9 muestran redundancia.

Se realizó un mapa de personas e ítems para evaluar la capacidad de cada ítem para medir distintos niveles de actitud y para describir la distribución de actitud en la muestra de participantes. La precisión de la medición se evaluó por medio del gráfico de función de información 
de la prueba. Se verificó el cumplimiento de los supuestos de unidimensionalidad e independencia local. Se utilizó el software Winsteps ${ }^{\circledast}$ y la librería eRm del paquete R para este análisis. El estudio se aprobó por el Comité de Ética e Investigaciones de la Fundación Universitaria de Ciencias de la Salud y de la Clínica el Country de Colombia.

\section{RESULTADOS}

\section{Teoría clásica de prueba}

\section{Construcción de la escala}

La búsqueda de literatura permitió identificar la escala IIFAS $^{(9)}$ como el modelo a partir del cual se elaboraron 17 ítems relacionados con los temas presentados en la nueva escala de actitud hacia la lactancia, según el contexto cultural actual, estos son participación de la pareja, redes de apoyo social, lactancia como medio de comuni- cación entre madre e hijo, duración de la lactancia y relación entre la lactancia y la salud de la madre, los cuales se incorporaron para la construcción de la nueva escala.

Los ítems se construyeron en escala tipo Likert, con 5 opciones de respuesta:

1. Totalmente en desacuerdo

2. En desacuerdo

3. Ni de acuerdo ni en desacuerdo

4. De acuerdo

5. Totalmente de acuerdo.

Los ítems 2, 6, 14 y 17 se puntúan de manera inversa. Se consideraron dos posibles dimensiones de la actitud hacia la lactancia: conocimiento (medido por los ítems $1,2,3,6,8,10,13,15)$ y actitud (medida por los ítems $4,5,7,9,11,12,14,16,17)$. Los ítems de la escala, junto con los resultados de la validación semántica y la versión en español, se presentan en la Tabla 1.

Tabla 1. Validación semántica de los ítems de la escala

\begin{tabular}{|c|c|c|c|c|}
\hline Ítem & Suficiencia & Coherencia & Relevancia & Claridad \\
\hline 1. Amamantar a mi bebé los primeros 6 meses de vida le dará una mejor salud & 4 & 4 & 4 & 4 \\
\hline $\begin{array}{l}\text { 2. Es más fácil controlar la alimentación de mi bebé con el tetero que cuando lo } \\
\text { amamanto }\end{array}$ & 3 & 3 & 3,5 & 3 \\
\hline 3. Mi bebé puede manifestarme cuando está lleno y no quiere tomar más leche & 3,5 & 4 & 4 & 4 \\
\hline 4. Para mí no tiene importancia el lugar donde doy pecho a mi bebé & 4 & 4 & 4 & 4 \\
\hline $\begin{array}{l}\text { 5. Se puede continuar la lactancia materna después de terminar la licencia de } \\
\text { maternidad }\end{array}$ & 4 & 4 & 4 & 4 \\
\hline 6. La lactancia prolongada luego de 6 meses afecta mi salud & 4 & 4 & 4 & 4 \\
\hline 7. El apoyo en el proceso de la lactancia por mi pareja es importante para mí & 4 & 4 & 4 & 4 \\
\hline 8. La lactancia materna es menos costosa que la lactancia con leche de fórmula & 2,5 & 3 & 3,5 & 3,5 \\
\hline $\begin{array}{l}\text { 9. Siento menos satisfacción personal cuando alimento a mi bebé con leche de } \\
\text { fórmula infantil que con leche materna }\end{array}$ & 4 & 4 & 4 & 4 \\
\hline 10. La forma como me alimento afecta la calidad de la leche que produzco & 3,5 & 4 & 4 & 4 \\
\hline 11. La actitud de mi pareja en relación con la lactancia es muy importante para mí & 4 & 4 & 4 & 4 \\
\hline 12. Extraer mi leche es otra opción de alimentar con pecho a mi bebé & 3,5 & 4 & 4 & 3,5 \\
\hline 13. Mi bebé crece mejor si lo alimento solo con el pecho & 3,5 & 3,5 & 3,5 & 4 \\
\hline 14. Amamantar a mi bebé en forma permanente afecta mi figura & 3,5 & 4 & 4 & 3,5 \\
\hline 15. Mi bebé tiene más riesgo de engordarse si lo alimento con fórmula infantil & 3,5 & 3 & 4 & 4 \\
\hline $\begin{array}{l}\text { 16. Mi bebé acepta más fácil el inicio de la alimentación complementaria cuando } \\
\text { se alimenta con pecho que por fórmula infantil }\end{array}$ & 4 & 4 & 4 & 4 \\
\hline 17. La leche de fórmula infantil es igual de saludable a la leche materna & 3,5 & 3,5 & 3,5 & 4 \\
\hline
\end{tabular}




\section{Validación de la escala}

En el estudio de validación se incluyeron 518 madres. La mediana de edad fue 28 años (rango intercuartílico (RIQ) 23,4 a 34). La mayoría de las entrevistadas eran casadas, con $1(246,47,6 \%)$ o 2 hijos (196, 37,9\%), escolaridad baja o mediana (436, $84.4 \%$ ) y con tiempo de regreso al trabajo programado en más de 3 meses (203, 60.8\%). El parto por cesárea estuvo en el 55,4\% de los casos. Los niños se encontraban dentro del rango normal en peso, talla y edad gestacional, como se observa en la Tabla 2.

Tabla 2. Características de las participantes en el estudio de validación

\begin{tabular}{|c|c|}
\hline Variable $^{1}$ & N (\%) \\
\hline Edad & $28(23,4-34)$ \\
\hline $\begin{array}{l}\text { Hijos } \\
-1 \\
-2 \\
-3 \text { o más }\end{array}$ & $\begin{array}{l}246(47,6) \\
196(37,9) \\
75(14,5)\end{array}$ \\
\hline $\begin{array}{l}\text { Escolaridad } \\
\text { - Primaria } \\
\text { - Bachillerato } \\
\text { - Profesional } \\
\text { - Posgrado }\end{array}$ & $\begin{array}{l}27(5,2) \\
221(42,8) \\
215(41,6) \\
54(10,4)\end{array}$ \\
\hline $\begin{array}{l}\text { Regreso al trabajo } \\
-\quad \text { Menos de } 1 \text { mes } \\
-1 \text { mes } \\
-2 \text { meses } \\
-3 \text { meses } \\
-\quad \text { Más de } 3 \text { meses }\end{array}$ & $\begin{array}{l}15(4,5) \\
3(0,9) \\
7(2,1) \\
106(31,7) \\
203(60,8)\end{array}$ \\
\hline Peso de la madre en kg & $69(62-78)$ \\
\hline Talla de la madre en $\mathrm{m}$ & $1,6(1,4-1,8)$ \\
\hline Peso del niño en g & $3010(2760-3300)$ \\
\hline Talla del niño en cm & $50(49-51)$ \\
\hline Sexo del niño, femenino & $262(50,6)$ \\
\hline Edad gestacional en semanas & $38(38-39)$ \\
\hline Vía del parto, cesárea & $285(55,4)$ \\
\hline
\end{tabular}

'Se presenta la mediana y el rango intercuartílico para las variables cuantitativas, y la frecuencia absoluta y relativa para las cualitativas.

\section{Validez de contenido}

La prueba de Bartlett (Ji cuadrado= 1249,4 (136), $p<0,001)$ y el criterio de Kaiser-Meyer-Olkin $(0,70)$ indicaron que la matriz de correlaciones era factoriza- ble. Aunque la extracción inicial por medio del método de factores principales y el gráfico de sedimentación sugirieron dos factores, se decidió considerar un solo factor, pues la solución sin rotar y las soluciones rotadas agruparon la mayoría de los ítems en el primer factor, lo que deja en el segundo los ítems de puntuación inversa, los cuales incluyeron ítems tanto para medir la actitud como el conocimiento (Tabla 3 ).

\section{Validez de constructo convergente}

La correlación de Spearman entre la escala de actitud hacia la lactancia y la IIFAS fue de 0,363 $(p<0,001)$. El resultado indica que la evaluación de la actitud hacia la lactancia materna mide un constructo asociado con lo evaluado por la IIFAS (el relacionado con las leches de fórmula), pero que difiere en otros aspectos, como la participación de la pareja, redes de apoyo social, lactancia como medio de comunicación entre madre e hijo, duración de la lactancia, relación entre la lactancia y la salud de la madre.

\section{Consistencia interna}

Se encontró un coeficiente alpha de Cronbach de 0,538; el cual no disminuyó en forma significativa si se retiraran ítems de la escala (datos no presentados).

\section{Confiabilidad del examen y repetición del examen}

El tiempo mediano entre aplicaciones de las escalas fue 8 días (rango intercuartílico 7 a 8 días, mínimo 4 días, máximo 11 días). El promedio de resultados obtenidos en la primera aplicación fue 71,6 $(\mathrm{DE}=5,2)$, y el promedio para la segunda fue $72,0(\mathrm{DE}=4,3)$. El coeficiente rho de Lin fue 0,377 (IC 95\% 0,140-0,614). La diferencia entre las dos aplicaciones fue $-0,44$ y los límites de Bland y Altman estuvieron entre -10,8 y 9,9.

\section{Teoría de respuesta al ítem}

La matriz de datos cumplió con los criterios de unidimensionalidad (prueba de Martin Löf: Ji cuadrado: 572,745; $p=1,000$ ). En la Tabla 4 se presentan los estadísticos de ajuste de los ítems. Las MNSQ estuvieron dentro de los rangos esperados, mientras que las ZSTD mostraron un caso de pobre ajuste ("para mí no tiene importancia el lugar donde doy seno a mi bebé”); sin embargo, se conserva esta pregunta dado que, en su versión sin estandarizar, los índices son adecuados. 
Tabla 3. Cargas factoriales para los ítems de la escala para la evaluación de la actitud hacia la lactancia materna

\begin{tabular}{|c|c|c|c|}
\hline Ítems & Factor 1 & Factor 2 & Unicidad \\
\hline 1. Amamantar a mi bebé los primeros 6 meses de vida le dará una mejor salud & 0,572 & $-0,2738$ & 0,5978 \\
\hline 2. Es más fácil controlar la alimentación de mi bebé con el tetero que cuando lo amamanto & 0,2651 & 0,4906 & 0,6891 \\
\hline 3. Mi bebé puede manifestarme cuando está lleno y no quiere tomar más leche & 0,2564 & $-0,1481$ & 0,9123 \\
\hline 4. Para mí no tiene importancia el lugar donde doy pecho a mi bebé & 0,1166 & $-0,1311$ & 0,9692 \\
\hline 5. Se puede continuar la lactancia materna después de terminar la licencia de maternidad & 0,5616 & $-0,1812$ & 0,6518 \\
\hline 6. La lactancia prolongada luego de 6 meses afecta mi salud & 0,3515 & 0,6835 & 0,4093 \\
\hline 7. El apoyo en el proceso de la lactancia por mi pareja es importante para mí & 0,5458 & $-0,0109$ & 0,702 \\
\hline 8. La lactancia materna es menos costosa que la lactancia con leche de fórmula & 0,6047 & $-0,1196$ & 0,6201 \\
\hline $\begin{array}{l}\text { 9. Siento menos satisfacción personal cuando alimento a mi bebé con leche de fórmula } \\
\text { infantil que con leche materna }\end{array}$ & 0,4137 & $-0,0552$ & 0,8258 \\
\hline 10. La forma como me alimento afecta la calidad de la leche que produzco & 0,4161 & $-0,299$ & 0,7375 \\
\hline 11. La actitud de mi pareja en relación con la lactancia es muy importante para mí & 0,5551 & $-0,2359$ & 0,6363 \\
\hline 12. Extraer mi leche es otra opción de alimentar con pecho a mi bebé & 0,4671 & $-0,2817$ & 0,7024 \\
\hline 13. Mi bebé crece mejor si lo alimento solo con el pecho & 0,546 & $-0,0231$ & 0,7014 \\
\hline 14. Amamantar a mi bebé en forma permanente afecta mi figura & 0,3027 & 0,597 & 0,5519 \\
\hline 15. Mi bebé tiene más riesgo de engordarse si lo alimento con fórmula infantil & 0,124 & 0,0298 & 0,9837 \\
\hline $\begin{array}{l}\text { 16. Mi bebé acepta más fácil el inicio de la alimentación complementaria cuando se alimenta } \\
\text { con pecho que por fórmula infantil }\end{array}$ & 0,0226 & $-0,0634$ & 0,9955 \\
\hline 17. La leche de fórmula infantil es igual de saludable a la leche materna & 0,3836 & 0,6751 & 0,3971 \\
\hline
\end{tabular}

El mapa de personas e ítems (Figura 1) mostró la distribución de las personas entre -2 y 3 logits, siendo más frecuente entre 0 y 2 logit. Como una posición más alta en el mapa muestra mayor actitud, la distribución encontrada indica que las participantes tuvieron, en su mayoría, una actitud promedio a alta. La distribución de los ítems fue más estrecha, entre -1 y casi 1 logit, lo que indica que la escala cuenta con ítems para medir un nivel intermedio de actitud. Como es mayor la media para personas que ítems, se sugiere que la muestra de participantes tenga una actitud mayor que la que obtendría una muestra aleatoria de participantes a quienes se aplica la escala. El ítem 15 ("mi bebé tiene más riesgo de engordarse si lo alimento con fórmula infantil”) está en una posición superior, siendo así un buen marcador para la medición de la actitud. Se observó un vacío de ítems para la discriminación entre los niveles muy altos de actitud. Este resultado es consistente con los índices de confiabilidad y de separación: 0,63 y 1,30 para personas y 0,98 y 7,33 para los ítems, lo que indica que la escala puede carecer de ítems para discriminar ciertos niveles del constructo.

Las gráficas mostradas en la Figura 2 muestran las distintas opciones de respuesta en relación con el nivel de actitud que miden, puede observarse, en general, cuáles opciones de respuesta mayores se asocian con un mayor constructo; sin embargo, en la mayoría de ítems, el rango del constructo se abarca por 3 o 4 opciones de respuesta.

La función de información de los ítems y de la prueba se pueden observar en la Figura 3. Esta gráfica indica que, si bien la prueba mide la actitud de las personas en un amplio rango del constructo, las mediciones más precisas se producen en el centro del mismo, entre -2 a 2 logits, lo que indica que la escala puede arrojar más información en niveles medios de actitud. 
Tabla 4. Estadísticos de ajuste de los ítems según la teoría de respuesta al ítem

\begin{tabular}{|c|c|c|c|c|}
\hline \multirow[t]{2}{*}{ Ítem } & \multicolumn{2}{|c|}{ INFIT } & \multicolumn{2}{|c|}{ OUTFIT } \\
\hline & MNSQ & ZSTD & MNSQ & ZSTD \\
\hline 1. Amamantar a mi bebé los primeros 6 meses de vida le dará una mejor salud & 0,95 & $-0,2$ & 0,83 & $-0,5$ \\
\hline 2. Es más fácil controlar la alimentación de mi bebé con el tetero que cuando lo amamanto & 0,96 & $-0,7$ & 0,96 & $-0,5$ \\
\hline 3. Mi bebé puede manifestarme cuando está lleno y no quiere tomar más leche & 1,08 & 1,1 & 1,11 & 1,5 \\
\hline 4. Para mí no tiene importancia el lugar donde doy pecho a mi bebé & 1,25 & 5,0 & 1,46 & 7,2 \\
\hline 5. Se puede continuar la lactancia materna después de terminar la licencia de maternidad & 0,95 & $-0,4$ & 1,0 & 0,0 \\
\hline 6. La lactancia prolongada luego de 6 meses afecta mi salud & 0,91 & $-1,3$ & 0,89 & $-1,3$ \\
\hline 7. El apoyo en el proceso de la lactancia por mi pareja es importante para mí & 0,92 & $-1,0$ & 0,95 & $-0,5$ \\
\hline 8. La lactancia materna es menos costosa que la lactancia con leche de fórmula & 0,95 & $-0,4$ & 0,86 & $-1,0$ \\
\hline $\begin{array}{l}\text { 9. Siento menos satisfacción personal cuando alimento a mi bebé con leche de fórmula } \\
\text { infantil que con leche materna }\end{array}$ & 0,99 & $-0,1$ & 1,04 & 0,6 \\
\hline 10. La forma como me alimento afecta la calidad de la leche que produzco & 1,07 & 0,9 & 1,14 & 1,5 \\
\hline 11. La actitud de mi pareja en relación con la lactancia es muy importante para mí & 0,98 & $-0,3$ & 0,98 & $-0,2$ \\
\hline 12. Extraer mi leche es otra opción de alimentar con pecho a mi bebé & 0,98 & $-0,2$ & 1,02 & 0,3 \\
\hline 13. Mi bebé crece mejor si lo alimento solo con el pecho & 0,98 & $-0,1$ & 0,95 & $-0,5$ \\
\hline 14. Amamantar a mi bebé en forma permanente afecta mi figura & 0,96 & $-0,6$ & 0,94 & $-0,9$ \\
\hline 15. Mi bebé tiene más riesgo de engordarse si lo alimento con fórmula infantil & 1,11 & 2,2 & 1,13 & 2,4 \\
\hline $\begin{array}{l}\text { 16. Mi bebé acepta más fácil el inicio de la alimentación complementaria cuando se alimenta } \\
\text { con pecho que por fórmula infantil }\end{array}$ & 1,15 & 2,4 & 1,17 & 2,7 \\
\hline 17. La leche de fórmula infantil es igual de saludable a la leche materna & 0,85 & $-2,2$ & 0,79 & $-2,6$ \\
\hline
\end{tabular}

MNSQ: show mean-square; ZSTD standardized as a z-score

\section{DISCUSIÓN}

Dada la importancia de la actitud hacia la lactancia materna, este estudio propone una nueva escala de medición de la actitud hacia la lactancia, presenta su proceso de desarrollo y una primera validación por medio de la teoría clásica de prueba y la teoría de respuesta al ítem.

La experiencia previa al emplearla evidenció elementos contextuales, que dieron motivo para diseñar una nueva escala en nuestro medio(14). La escala $\operatorname{IIFAS}^{(8)}$, en su diseño básico original, hace un énfasis importante en determinar la actitud de la lactancia frente al uso de sucedáneos de la leche materna. Asimismo, posee conceptos relacionados con el momento cultural y social de la década de 1990, que describe la percepción del hombre hacia la lactancia bajo un término cultural de género, la polémica frente al consumo de bebidas alcohólicas y su relación con la lactancia.

Sin perder vigencia, los elementos que conforman los constructos requieren de un cambio de contexto acorde a la cultura y sociedad actual. Hoy en día hay elementos que inciden en el éxito o fracaso de una mujer hacia la lactancia exclusiva, como la participación de las redes sociales y su papel de apoyo a la lactancia, la existencia y cooperación de grupos de apoyo, la participación de la pareja y amigos, e incluso el concepto nutricional que desempeña la lactancia frente al uso de sucedáneos de la leche materna y el riesgo de obesidad infantil ${ }^{(17,18)}$.

La población predominante del presente estudio estuvo conformada por madres con un estrato social medio a medio bajo, con nivel educativo bajo (solo bachillerato), en su mayoría jóvenes y con una relación 


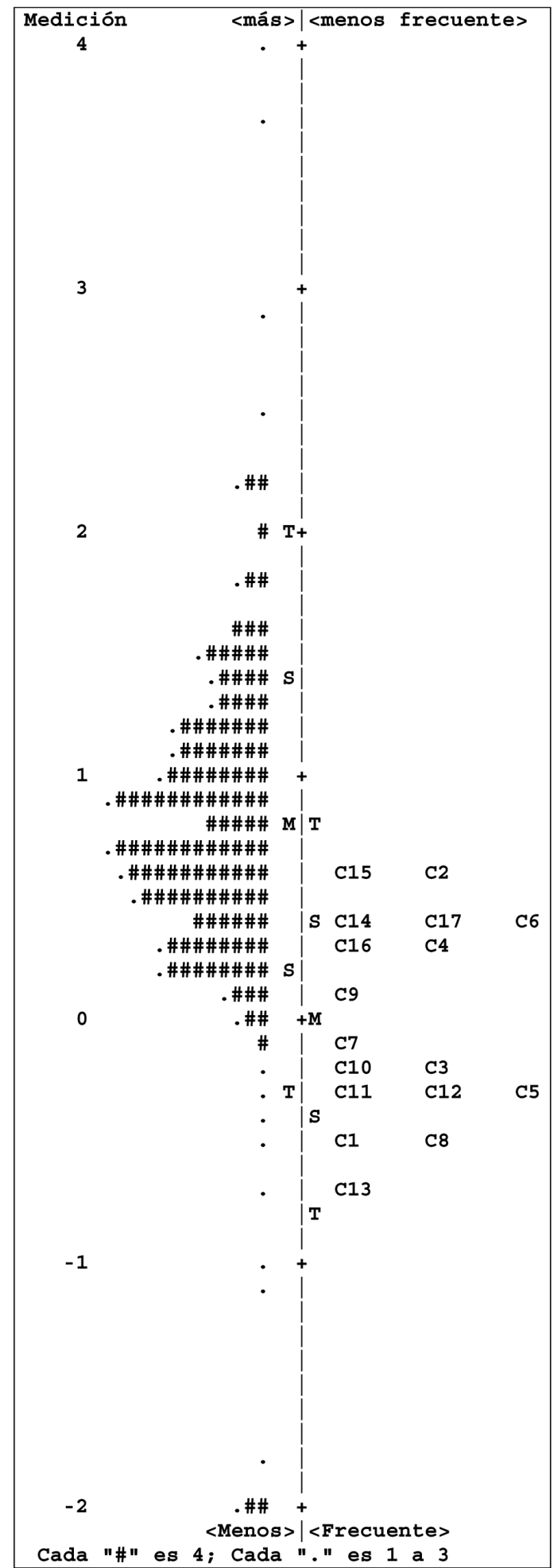

Figura 1. Mapa de personas e ítems de Wright de la escala para la evaluación de actitud hacia la lactancia materna. Izquierda: cada "\#" corresponde a 4 pacientes, y mide la distribución de actitud frente a las respuestas (logit = probabilidad de respuesta). Derecha: corresponde a la distribución de las preguntas de acuerdo con la actitud. La altura en la gráfica corresponde a la magnitud de la actitud; esta va de menor a mayor. marital. Esta población es representativa de madres lactantes colombianas, en quienes se pueden llevar a cabo programas de fortalecimiento a la lactancia, similar a lo desarrollado en otros estudios de evaluación de actitud o autoeficacia hacia la lactancia ${ }^{(12,19-22)}$.

El análisis de confiabilidad arrojó bajos coeficientes, tanto de consistencia interna como de concordancia y correlación de Lin, ello es posible en las condiciones de aplicación, dado que las madres respondieron la escala en el puerperio inmediato, momento de estrés ${ }^{(23)}$, teniendo en cuenta que la segunda aplicación se realizó cuando ya se encontraban fuera de las instituciones y tenían un tiempo de experiencia con el proceso de la lactancia. Pensamos que puede mejorar la estimación de la confiabilidad, con la aplicación de la escala en otras circunstancias, por ejemplo, durante el embarazo, momento clave para identificar a las mujeres que requerirán algún tipo de consejería con la lactancia. La correlación moderada entre la escala IIFAS y la colombiana mostró aspectos culturales que difieren en el desarrollo del constructo de actitud.

El análisis factorial exploratorio sugirió dos posibles factores, sin embargo, el análisis del contenido del segundo factor permitió observar que se agruparon los ítems redactados de manera inversa y que corresponden a aspectos de actitud y conocimiento, por lo que se consideró un solo factor subyacente.

En el análisis de la escala mediante la teoría de respuesta al ítem, los resultados obtenidos mostraron que la escala tiene ítems que discriminan bien la actitud hacia la lactancia en participantes con niveles de actitud media y baja. La muestra de que se obtuvo la validación incluyó participantes con niveles de actitud medios a altos, como se observa en el mapa de personas e ítems (Figura 1), siendo una población homogénea en cuanto a actitud. Se considera que esta homogeneidad se atribuye a que la población estuvo constituida principalmente por madres de estratos medios y medios bajos. Aunque se conoció el nivel educativo de la muestra, no se evaluaron otros aspectos culturales, que permitieran definir mejor la razón de esta homogeneidad.

$\mathrm{Al}$ analizar el comportamiento en cada una de las preguntas en las estadísticas de ajuste, hay preguntas, como la 6 y 17, que demostraron redundancia. Otras preguntas tienen una redacción similar a estos ítems: por ejemplo, la pregunta 6 ("la lactancia prolongada mayor de 6 meses afecta mi salud"), puede relacionarse con la 14 ("amamantar a mi bebé en forma permanente afecta mi figura"). Al analizar el detalle de su significado, encontramos que la pregunta 14 hace referencia a un 

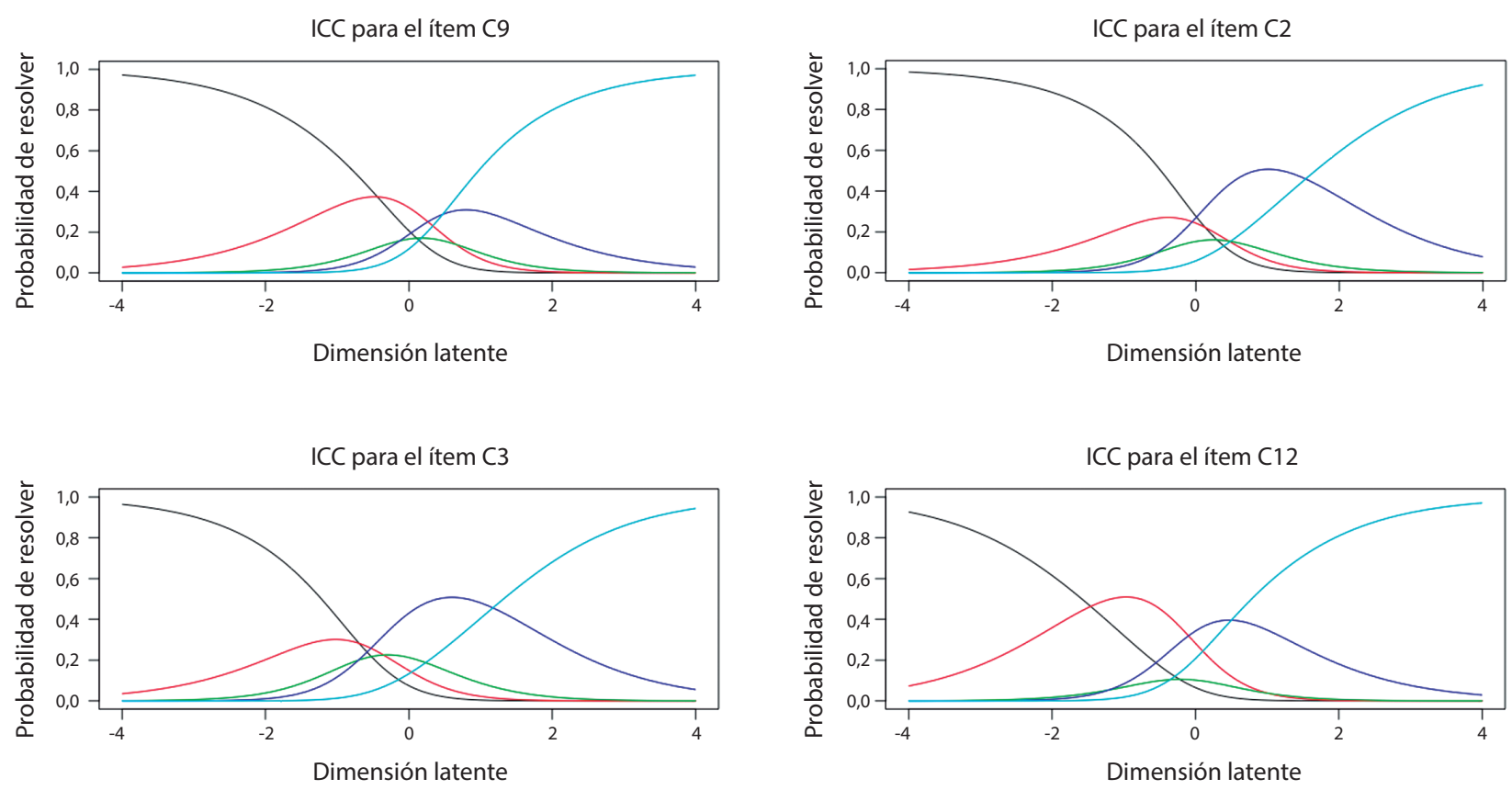

Figura 2. Evaluación de la escala de medición. Ejemplo para los ítems 2, 3, 9 y 12. ICC: curva característica del ítem (Item Characteristic Curve)
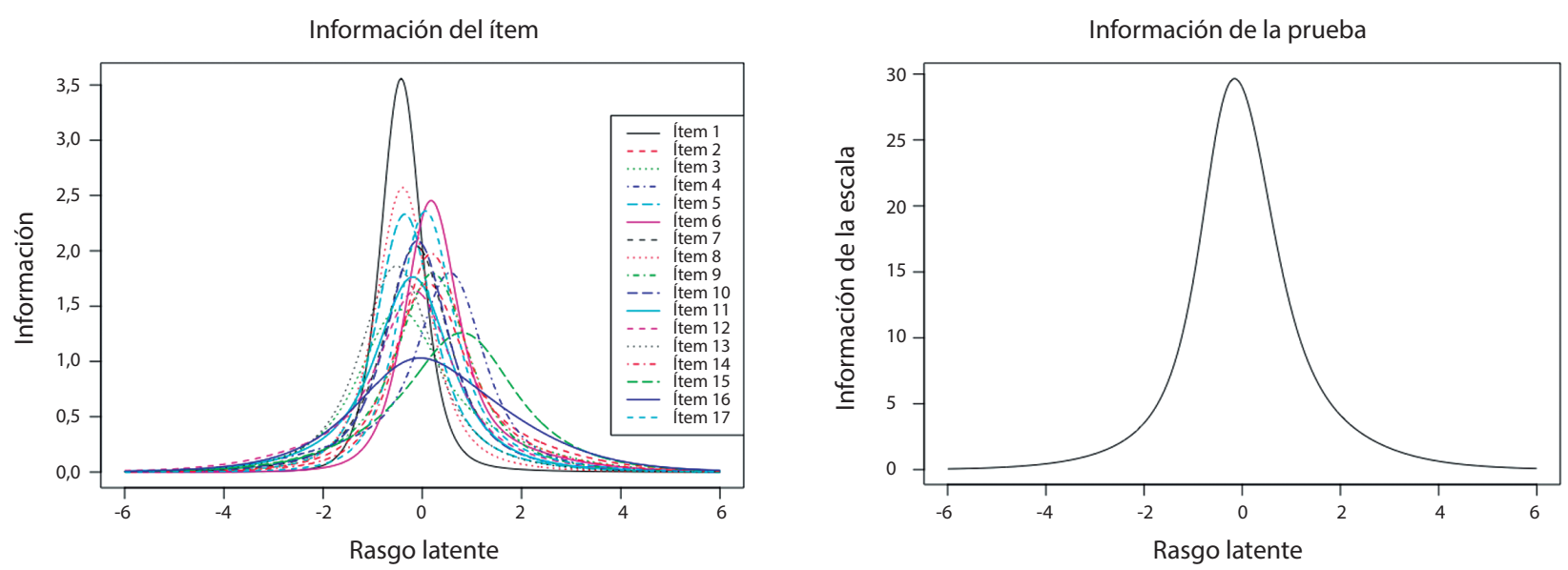

Figura 3. Función de información de la prueba.

aspecto estético, mientras que la pregunta 6 cuestiona un tema relacionado con la salud como consecuencia de la lactancia prolongada. En temas como el peso y el estado nutricional de la madre, ambas preguntas midieron igual cantidad de actitud, por lo que se conservaron en la escala.

El ítem 17 ("la leche de fórmula infantil es igual de saludable a la leche materna") puede relacionarse con el 15 ("mi bebe tiene más riesgo de engordarse si lo ali- mento con fórmula infantil"). Las dos preguntas tienen conceptos diferentes, la pregunta 17 se refiere al conocimiento por parte de la mujer en relación con la composición nutricional y biológica de ambas leches y los resultados biológicos y nutricionales en el uso de estas. La pregunta 15 está orientada al riesgo de obesidad con el uso de sucedáneos de la leche con respecto a la leche materna; por lo anterior, estas preguntas se mantuvieron en la escala final. Es interesante anotar que la pre- 
gunta 15 tuvo una localización en el mapa de personas e ítems en la parte superior, lo que permite discriminar alta actitud hacia la lactancia.

$\mathrm{Al}$ analizar las diferencias entre las preguntas que tienen mayor probabilidad de respuesta (fáciles), frente a aquellas catalogadas como difíciles, la facilidad de las primeras se atribuye a la claridad de los temas en relación con el constructo al momento actual: apoyo de la pareja, lactancia extendida, extracción de la leche para el trabajo, resultado óptimo en la salud del niño; en cambio, las preguntas que están catalogadas como difíciles, aquellas con puntuación inversa cuya respuesta negativa significa una actitud o conocimiento positivo frente a la lactancia, implican un conocimiento más profundo respecto a la lactancia en la persona: riesgo de obesidad en el lactante, riesgo de cambios nutricionales en la mujer y facilidad de inicio de la dieta complementaria.

En cuanto al sistema de puntuación Likert de la escala, se observaron puntuaciones que no se están utilizando por las pacientes, lo que sugiere que podría emplearse un número menor de niveles: muy en desacuerdo, neutro y muy de acuerdo. Estas opciones son adecuadas, teniendo en cuenta que el empleo de la totalidad de las opciones de respuesta puede llevar a los encuestados a elegir opciones de indiferencia, por tanto, no se alcanzaría la medición adecuada del constructo ${ }^{(23,24)}$.

En relación con las fortalezas del trabajo, tenemos la evaluación de la escala con dos metodologías: la teoría clásica de prueba y la metodología de la teoría de respuesta al ítem. Esta última tiene ventajas frente a la primera en la validación de escalas al aportar análisis a nivel de ítem, evaluar la capacidad de la escala para discriminar distintos niveles del constructo y no depender de la población en la que se aplica la escala para obtener las estimaciones ${ }^{(24)}$. Entre las limitaciones del estudio se puede mencionar la mayor proporción de personas con una distribución social media y media baja, con un bajo nivel educativo, en relación con la capacidad de respuesta y con la selectividad en las posibilidades de respuesta, por lo que se plantea la necesidad de aplicación en una población más heterogénea.

\section{CONCLUSIONES}

En conclusión, el empleo de la escala en actitud hacia la lactancia materna es válida para discriminar el constructo en pacientes con niveles bajos y medios, mostrando su utilidad para identificar a las madres que se beneficiarían de intervenciones para mejorarla. Se necesita la inclusión de ítems para discriminar niveles altos. Asimismo, es importante impulsar la aplicación de la escala en diversas poblaciones, con objeto de conocer el comportamiento de esta, y así poder ajustarla más.

\section{PUNTOS CLAVE}

- Escala psicométrica para medir la actitud materna frente a la lactancia

- Desarrollo y evaluación de la escala bajo dos metodologías

- Actualización de la escala de acuerdo con parámetros sociales actuales.

\section{Agradecimientos}

Agradecemos a Helen Preciado Barrera, del departamento de Pediatría de la Fundación Universitaria de Ciencias de la Salud, Hospital de San José de Bogotá; a Mayra Ivonne Ramírez, residente de tercer año; y a Laura del Pilar Villarreal Torres, residente de segundo año de Pediatría, ambas de la Fundación Universitaria de Ciencias de la Salud, Hospital de San José de Bogotá.

\section{Financiamiento}

El presente trabajo no contó con financiación.

\section{Conflicto de intereses}

Los autores declaran no tener conflicto de intereses en la realización del presente trabajo.

\section{Declaración de autoría}

AJ diseñó la escala, la aplicación en una de las instituciones y participó activamente en el análisis y desarrollo del escrito. FS participó activamente en el análisis de los datos y en el desarrollo del escrito. LV participó activamente en la recolección de datos en una de las instituciones y en el análisis de los datos.

\section{Referencias bibliográficas}

1. Lactancia materna exclusiva [Internet]. Organización Mundial de la Salud. (Consultado el 12 de marzo de 2021). Disponible en: https://www.who.int/nutrition/topics/exclusive_breastfeeding/es/

2. Martínez AMR, Hernández LMJ, Hernández LMV. Psicometría. Madrid: Alianza Editorial. 2014.

3. Crocker LM, Algina J. Introduction to classical and modern test theory. New York: Holt, Rinehart, and Winston. 1986. 
4. DeVellis RF. Scale development: theory and applications. $3^{a}$ edición. California: SAGE Publications. 2012. p. 73-114.

5. Steiner DL, Norman GR, Cairney J. Health measurement scales: A practical guide to their development and use. $5^{\text {a }}$ edición. Oxford: Oxford University Press. 2014.

6. Wolfe EW, Smith EV Jr. Instrument development tools and activities for measure validation using Rasch models: Part I - instrument development tools. J Appl Meas. 2007;8(1):97123.

7. Yang FM, Kao ST. Item response theory for measurement validity. Shanghai Arch Psychiatry. 2014;26(3):171-7. doi: 10.3969/j.issn.1002-0829.2014.03.010.

8. Kelley MA, Kviz FJ, Richman JA, Kim JH, Short C. Development of a scale to measure gender-role attitudes toward breast-feeding among primiparas. Women Health. 1993;20(1):47-68. doi: 10.1300/J013v20n01_04.

9. de la Mora A, Russell DW, Dungy CI, Losch M, Dusdiecker L. The Iowa Infant Attitude Scale: Analysis of reliability and validity. J Appl Soc Psychol. 1999;29(11):2362-80. doi: 10.1111/j.1559-1816.1999.tb00115.x.

10. Aluş TM, Okumuş H, Dennis CL. Translation and psychometric assessment of the Breast-feeding Self-Efficacy Scale-Short Form among pregnant and postnatal women in Turkey. Midwifery. 2010;26(1):101-8. doi: 10.1016/j. midw.2008.04.002.

11. Dencker A, Taft C, Bergqvist L, Lilja H, Berg M. Childbirth experience questionnaire (CEQ): Development and evaluation of a multidimensional instrument. BMC Pregnancy Childbirth. 2010;10:81. doi: 10.1186/1471-2393-10-81.

12. Holbrook KE, White MC, Heyman MB, Wojcicki JM. Maternal sociodemographic characteristics and the use of the Iowa infant attitude feeding scale to describe breastfeeding initiation and duration in a population of urban, Latina mothers: A prospective cohort study. Int Breastfeed J. 2013;8(1):7. doi: 10.1186/1746-4358-8-7.

13. Aguilar-Navarro HJ, Coronado-Castilleja A, GómezHernández OJ, Cobos-Aguilar H. Adaptación de la Iowa Infant Feeding Attitude Scale en población mexicana. Acta Pediatr Mex. 2016;37(3):149-58.
14. Jacome A, Jiménez R. Validación de la Iowa Infant Feeding Attitude Scale. Pediatr. 2014;47(4):77-82. doi: 10.1016/ S0120-4912(15)30143-9.

15. Matas A. Diseño del formato de escalas tipo Likert: un estado de la cuestión. REDIE. 2018;20(1):38-47. doi: 10.24320/ redie.2018.20.1.1347.

16. López PJA. Análisis psicométrico de la escala de marcha y equilibrio de Tinetti con el modelo de Rasch. Fisioterapia. 2009;31(5):192-202. doi: 10.1016/j.ft.2008.10.004.

17. Hancox RJ, Stewart AW, Braithwaite I, Beasley R, Murphy $\mathrm{R}$, Mitchell EA, et al. Association between breastfeeding and body mass index at age 6-7 years in an international survey. Pediatr Obesit. 2015;10(4):283-87. doi: 10.1111/ijpo.266.

18. Hansstein FV. The impact of breastfeeding on early childhood obesity: Evidence from the National Survey of Children. Am J Health Promot. 2016;30(4):250-8. doi: $10.1177 / 0890117116639564$.

19. Janke JR. Development of the breast-feeding attrition prediction tool. Nurs Res. 1994;43(2):100-4.

20. Dennis CL. The breastfeeding self-efficacy scale: Psychometric assessment of the short form. J Obstet Gynecol Neonatal Nurs. 2003;32(6):734-44. doi: 10.1177/0884217503258459.

21. Dennis CLE. Identifying predictors of breastfeeding self-efficacy in the immediate postpartum period. Res Nurs Health. 2006;29(4):256-68. doi: 10.1002/nur.20140.

22. Tuthill EL, McGrath JM, Graber M, Cusson RM, Young SL. Breastfeeding self-efficacy: A critical review of available instruments. Journal Hum Lact. 2016;32(1):35-45. doi: $10.1177 / 0890334415599533$.

23. Chabbert M, Guillemot-Billaud A, Rozenberg P, Wendland J. Determinants of anxiety symptoms, depression and peritraumatic distress in immediate postpartum women's mental health. Gynecol Obstet Fertil Senol. 2021;49(2):97-106. doi: 10.1016/j.gofs.2020.10.002.

24. Morales VP. Medición de actitudes en psicología y educación. 3a edición. Madrid: Universidad Ponticia Comillas. 2006. 